\title{
Effectiveness of Modified Early Warning Score (MEWS) in the Outcome of In-Hospital Adult Cardiac Arrests in a Tertiary Hospital
}

\section{Saumy Johnson ${ }^{1 *}$ and Anitha Nileswar}

${ }^{1}$ Department of Respiratory Therapy, Manipal University, Manipal 576104, Karnataka, India

${ }^{2}$ Department of Anesthesiology, KMC, Manipal University, Manipal 576104, Karnataka, India

\begin{abstract}
Aim: Aim of the present study was to evaluate the effectiveness of Modified Early Warning Score (MEWS) in the outcome of In- hospital adult cardiac arrest in a tertiary hospital.

Methodology: Study design was prospective interventional study, the study duration was from September 2009 to May 2013. Location was Kasturba Hospital, Manipal and study population was patients who sustained cardiac arrests inside the hospital. Tools used were Modified Utstein style Format for standard reporting of In-hospital cardiopulmonary resuscitation and Modified early warning score chart.
\end{abstract}

Inclusion criteria: Patients with Age $>18$ years and patients who sustained cardiac arrest

Exclusion criteria: Cardiac arrest in the operation theatre and Patients who were brought dead

Keywords: Empyema; Nosocomial; Pleural; Infection; Antibiotics; Co-amoxiclav; Thoracocentesis

\section{Introduction}

Cardiac arrest in a hospital often follows a period of severe illness and if the pre-arrest scenario is recognized early, cardiac arrest may be averted or identified in time. This should be reflected by an improvement in the outcome. The modified early warning score (MEWS) is a useful tool for identifying hospitalized patients in need of a higher level of care and those at risk of in-hospital death. Use of the MEWS as a triage tool to identify patients those who need hospital admission and those at increased risk of in-hospital death has been evaluated only to a limited extent [1]. Return of spontaneous circulation alone is not an indicator of successful resuscitation. Resuscitation can be deemed successful only if the victim survives to hospital discharge and returns to a reasonable quality of life.

MEWS, specifically five selected parameters, may be used as a rapid, simple triage method to identify medical patients in need of hospital admission and those at increased risk of in-hospital death [2]. Earlier recognition and treatment will revert the worsening of physiological parameters and enable judicious transfer to the intensive care unit for additional and more intensive support [3]. Most of the physiological scoring systems are scored by providing points to the observed values which include pulse rate, blood pressure, and respiratory rate. The final score is obtained by adding up each of the individual scores. Action plan for the score is provided to the staff. Early warning score tool has been evolved scientifically and it is a very important tool to determine the changes in the condition of the patient early so that it can be corrected at an earlier stage.

\section{Statistical Analysis}

Statistical analysis was performed using statistical software SPSS Version 16.0. Values are expressed in Mean \pm SD. Continuous variables were tested using Paired $t$ test and student $t$ test. Categorical variables were tested using chi square test. $\mathrm{P}$ value of $<0.05$ was considered significant (Flow Chart 1).

\section{Results}

This was a prospective study conducted over a period of 20 months. A total of $1,18,968$ inpatients were admitted to this tertiary care hospital in South India out of 1955 patients sustained cardiac arrest Incidence of 16.4 per every 1000 admissions. Table 1 shows the age and gender distribution of the pre and post MEWS patients.

The ROSC was better in the post MEWS period is given in Figure 1. There were more patients in the post MEWS period that had ROSC after cardiac arrest. The initial rhythm as VF/VT was found to be better in the chance of survival of the patients. It is given in Figure 2.

There was more number of patients in the CPC 1-2 than in CPC 3-4 in Pre MEWS and Post MEWS period. Comparatively there was increased number of patients in CPC 1-2 in the Post MEWS period (Figure 3).

The results were analyzed to determine the comparative difference between the Pre MEWS and Post MEWS. There was statistically significant difference in survival to hospital discharge, alive at 6 months and also Independent at ADL (Figure 4).

\section{Discussion}

In the present study the survival to hospital discharge improved significantly from $5 \%$ to $16.82 \%$ pre and post MEWS. It is similar to the results of Nauman Naeem et al. where introduction of MEWS resulted in a better survival to hospital discharge and it was statistically significant [4]. Bloom et al. compared the survival after IHCA in terms of short term and long term. They found $6.6 \%$ patients to survive at the time of moving out from hospital [5]. Sandroni et al. reported survival to hospital discharge varies from $0 \%$ to $42 \%$, the most common range being between $15 \%$ and $20 \%$ [6]. The in-hospital mortality was found to be $70.6 \%$ in one study. Mortality was lower in hospitals which were teaching in nature, urban set up and hospitals which are large. This

*Corresponding author: Saumy Johnson, Associate Professor and Head, Department of Respiratory Therapy, Manipal University, Manipal 576104, Karnataka, India, E-mail: saumy.johnson@manipal.edu

Received April 29, 2015; Accepted August 18, 2015; Published August 24, 2015

Citation: Johnson S, Nileswar A (2015) Effectiveness of Modified Early Warning Score (MEWS) in the Outcome of In-Hospital Adult Cardiac Arrests in a Tertiary Hospital. J Pulm Respir Med 5: 285. doi:10.4172/2161-105X.1000285

Copyright: ( 2015 Johnson S, et al. This is an open-access article distributed under the terms of the Creative Commons Attribution License, which permits unrestricted use, distribution, and reproduction in any medium, provided the original author and source are credited. 


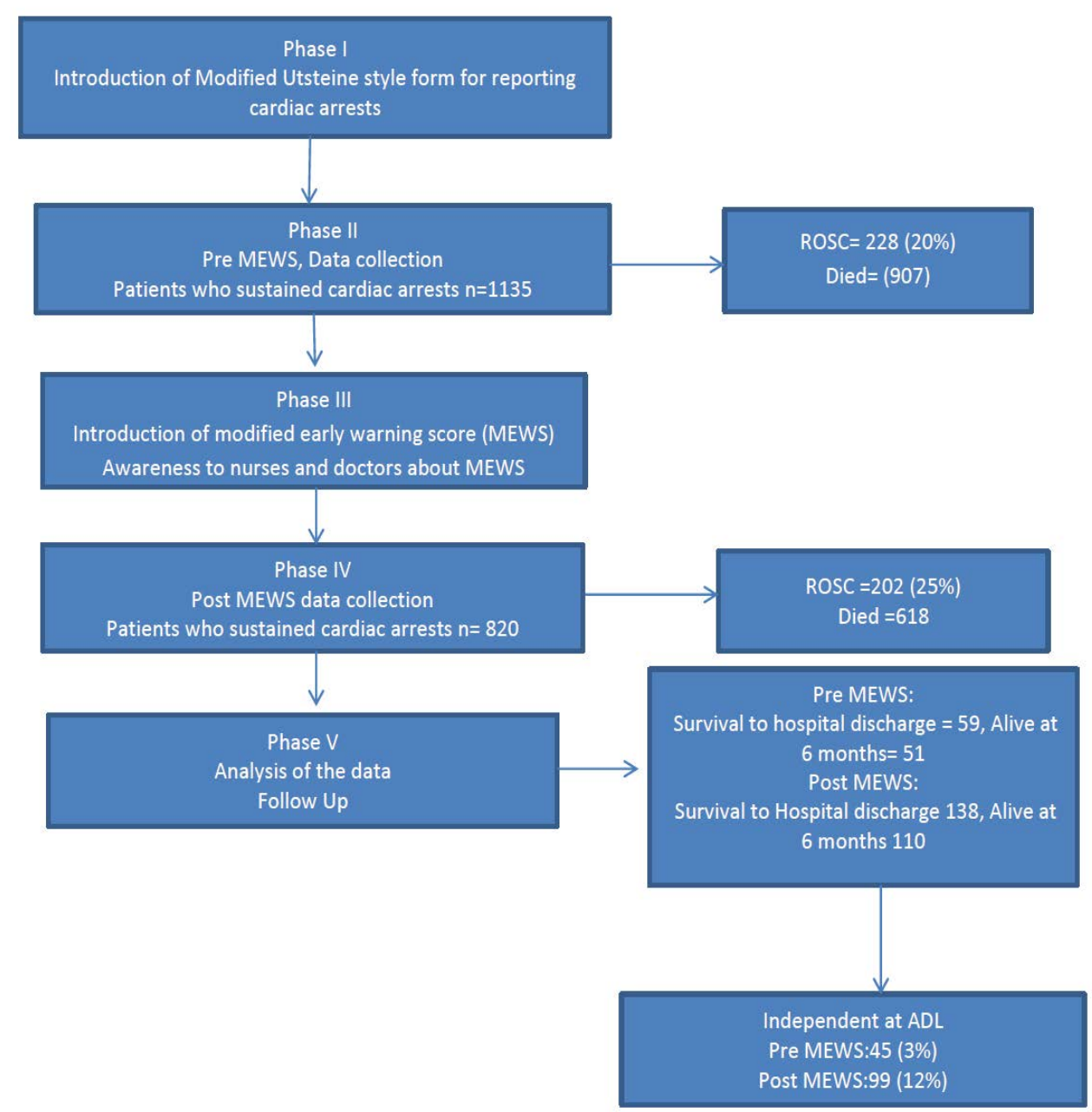

Flow Chart 1: Consort flow chart.

\begin{tabular}{|c|c|c|c|}
\hline & Pre MEWS & Post MEWS & P Value \\
\hline Age (Years) & $52.47 \pm 17.27$ & $53.72 \pm 16.84$ & $>0.05$ \\
\hline Gender M/F & $770 / 365$ & $557 / 263$ & $<0.05$ \\
\hline
\end{tabular}

study also established the implications for the propagation of finest treatments in the post resuscitation care [7].

There was an increase in the number of patients who had VF/VT after the introduction of MEWS in the present study (8.5\% vs. $23.7 \%$ ). Zubek et al. ROSC occurred in $58 \%$ of VF cases, yielding a survival tohospital discharge rate of $34 \%$ in this subset of patients [8].

In our study there was no significant difference in the CPC but there was a change in the ADL in the post MEWS period. This shows that when the patients were resuscitated earlier, the outcome both in terms of survival and neurological status in the long term is better.

This study suggest that MEWS could be widely used in the hospitals to detect the deterioration of in- patients by the ward nurse and the patients could be resuscitated effectively and quite earlier so that the neurological status is regained in a better manner

Girotra et al. in their study found that the survival and neurological outcome of the victims who sustained IHCA have improved over the years [9]. This is the one among the few studies that evaluated the effectiveness of MEWS in CPC and ADL. Wachelder et al. concluded in their study that when there was a person available for CPR who was not a member of the family, then the survival and neurological status was better. Long term survival in elderly in OHCA was depended on the bystander CPR and 1 month survival had a relation with the CPR done by the person who was available at the site of CPR [10]. In our present study the setting was In- hospital and in the post MEWS period there was an improvement in the time of first compression and the survival rate and activities of daily living was better.

In our study there was no significant difference in the CPC but there was a change in the ADL in the post MEWS period which shows that when the patients were resuscitated earlier, the outcome both in terms of survival and neurological status in the long term. This study suggest that MEWS could be widely used in the hospitals to detect the deterioration of in- patients by the ward nurse and the patients could be resuscitated effectively and quite earlier so that the neurological status is regained in a better way.

\section{Conclusion}

The use of MEWS permits earlier detection of impending cardiac 


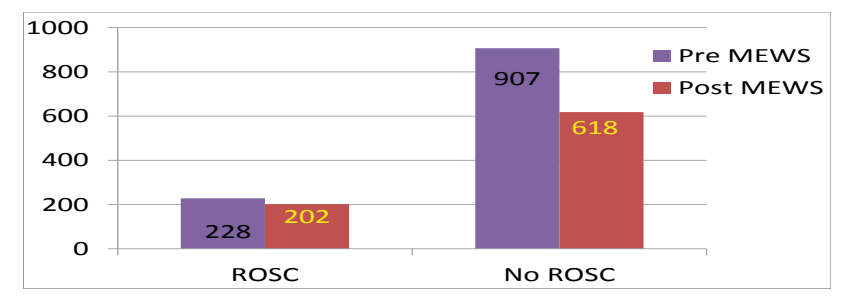

Figure 1: ROSC and non-ROSC.

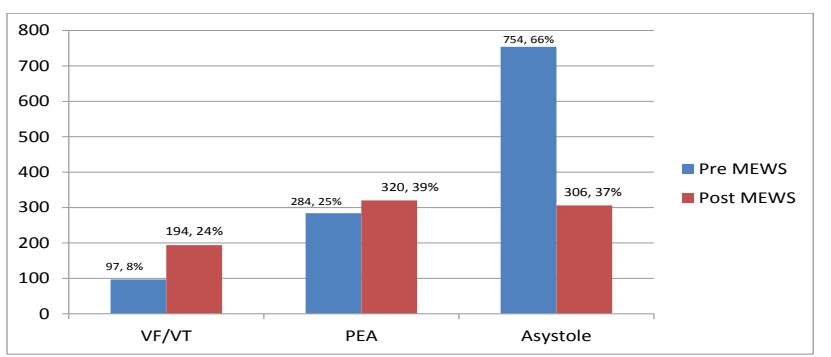

Figure 2: Initial rhythms of the patients.

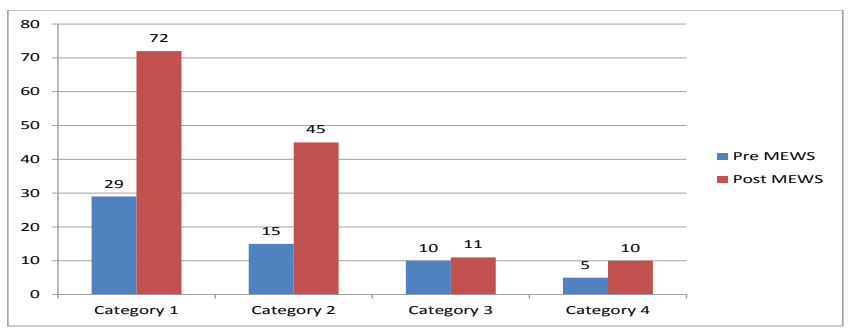

Figure 3: Cerebral performance category (CPC).

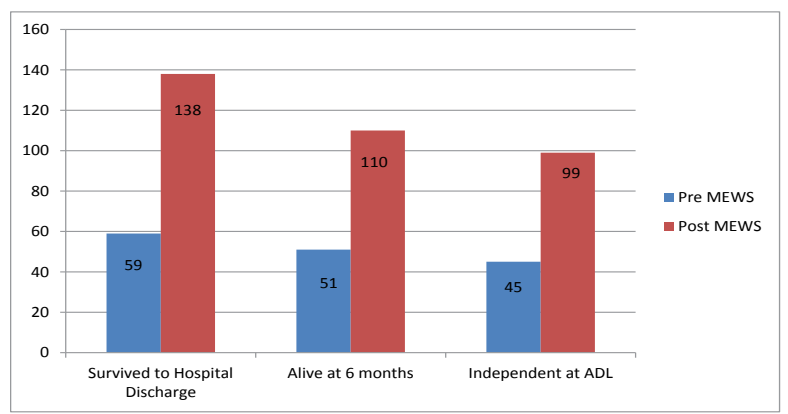

Figure 4: Survival at discharge, 6 months and independent in activities of daily living (ADL). arrests is associated with higher rates of VF/VT as the initial rhythm and better survival rates. The Quality of life is better for patients in the Post MEWS group in terms of Activities of Daily Living. There is a significant difference between the two groups in terms of sustaining of ROSC, Independence in activities of daily living and activities of daily living. MEWS could be widely used in patients in HDUs and wards.

\section{References}

1. Morgan RJM, Williams F, Wright MM (1997) An early warning scoring system for detecting developing critical illness. Clin Intensive Care 8: 100.

2. Burch VC, Tarr G, Morroni C (2008) Modified early warning score predicts the need for hospital admission and inhospital mortality. Emerg Med J 25: 674678.

3. Subbe CP, Kruger M, Rutherford $P$, Gemmel $L$ (2001) Validation of a modified Early Warning Score in medical admissions. QJM 94: 521-526.

4. Nauman Naeem. Beyond the intensive care unit: A review of interventions aimed at anticipating and preventing in-hospital cardiopulmonary arrest. Resuscitation 13-23.

5. Bloom HL, Shukrullah I, Cuellar JR, Lloyd MS, Dudley SC Jr, et al. (2007) Long-term survival after successful inhospital cardiac arrest resuscitation. Am Heart J 153: 831-836.

6. Sandroni C, Nolan J, Cavallaro F, Antonelli M (2007) In-hospital cardiac arrest: incidence, prognosis and possible measures to improve survival. Intensive Care Med 33: 237-245.

7. Carr BG, Goyal M, Band RA, Gaieski DF, Abella BS, et al. (2009) A national analysis of the relationship between hospital factors and post-cardiac arrest mortality. Intensive Care Med 35: 505-511.

8. Zubek L, Szabó L, Horváth L, Mesterházi A, Gál J, et al. (2012) Correlation between APACHE II score and quality of life among patients discharged from the ICU. Critical Care 16: P404.

9. Girotra S, Nallamothu BK, Spertus JA, Li Y, Krumholz HM, et al. (2012) Trends in survival after in-hospital cardiac arrest. N Engl J Med 367: 1912-1920.

10. Wachelder EM, Moulaert VR, van Heugten C, Verbunt JA, Bekkers SC, et al (2009) Life after survival: long-term daily functioning and quality of life after an out-of-hospital cardiac arrest. Resuscitation 80: 517-522. 